\title{
Internal reinstatement hides cuing effects in source memory tasks
}

\author{
Jeffrey J. Starns • Jason L. Hicks
}

Published online: 11 May 2013

(C) Psychonomic Society, Inc. 2013

\begin{abstract}
Reinstating source details at test often has no impact on source memory. We tested the proposition that participants internally reinstate source cues when such cues are not provided by the experimenter, thus making the external cues redundant. Participants studied words paired with either a male or a female face and were later asked to specify the gender of the face studied with each word. To disrupt the ability to internally reinstate sources, some participants saw eight male faces and eight female faces throughout the study list (multiple-face condition), making it difficult to determine which face should be internally reinstated for uncued test trials. Other participants saw only a single face for each gender (single-face condition), which should facilitate internal reinstatement. Across three experiments, participants in the multiple-face condition showed improved source discrimination when the studied faces were reinstated at test, as compared to uncued trials. In contrast, participants in the single-face condition showed no effect of the face cues. Moreover, the cuing effect for the multiple-face condition disappeared when the test structure facilitated internal reinstatement. Overall, the experiments support the contention that internal reinstatement is a natural part of source retrieval that can mask the effects of external cues.
\end{abstract}

Keywords Source memory $\cdot$ Reinstatement effects · Cuing effects

J. J. Starns

University of Massachusetts, Amherst, MA, USA

J. L. Hicks

Louisiana State University, Baton Rouge, LA, USA

J. J. Starns $(\bowtie)$

Department of Psychology, University of Massachusetts-Amherst,

441 Tobin Hall,

Amherst, MA 01003, USA

e-mail: jstarns@psych.umass.edu
An emphasis on the importance of retrieval cues permeates theories of long-term memory. General theoretical principles (e.g., encoding specificity; Tulving \& Thomson, 1973) and specific mathematical models of memory (e.g., global matching models; Clark \& Gronlund, 1996) have proposed that memory performance depends on the match between processing at encoding and retrieval. Retrieval cues should improve memory when they reinstate features of the encoding event.

Much cuing research has focused on the impact of reinstating contextual features (see Smith \& Vela, 2001, for a review). If contextual details are processed and incorporated into long-term memories at encoding (Johnson, Hashtroudi, \& Lindsay, 1993), then reinstating aspects of the encoding context at retrieval should improve memory performance. Indeed, recall consistently shows this benefit (Smith \& Vela, 2001). In a classic example, participants recall more words when they are tested in the same room in which they learned the words than when they are tested in a different room (e.g., Smith, 1979). In contrast, many experiments show no effect of context cues on discriminability in recognition and source tasks (Fernandez \& Glenberg, 1985; Godden \& Baddeley, 1980; Murnane \& Phelps, 1993, 1994, 1995; Smith, Glenberg, \& Bjork, 1978; Starns \& Hicks, 2005).

In this article, we focus on cuing effects in source memory (Johnson et al., 1993). In a source memory task, participants first study items in different contexts or presentation formats; for example, they might learn a list of words presented by a male or female speaker. Words are then presented at test, and participants are asked to identify the relevant source information (e.g., respond "male" or "female"). New items that were not encountered in any source are often included at test, in which case participants are required to both recognize words that they previously encountered and determine the source of each recognized word. Our primary goals are to test a theoretical explanation for null effects of source reinstatement and to develop procedures that promote consistent cuing effects. 


\section{Reinstatement effects in source memory}

A number of studies have evaluated reinstatement effects in source memory (Craik \& Kirsner, 1974; Dodson, 2007; Dodson \& Shimamura, 2000; Kirsner, 1973; Leynes, Bink, Marsh, Allen, \& May, 2003; Palmeri, Goldinger, \& Pisoni, 1993; Roediger, McDermott, Pisoni, \& Gallo, 2004; Starns \& Hicks, 2005; Vogt \& Bröder, 2007). In studies of this sort, the main question is whether reinstating source information improves memory for source. For example, an experiment might compare male/female source judgments for words played in the same voice at study and test versus words presented in a mismatching voice or with no voice information at test. This procedure seems to create an ideal situation for producing positive cuing effects, because the same source information that the participant must retrieve is made available in the test cue. Nevertheless, existing studies provide only limited evidence that source reinstatement improves source discrimination.

Several studies show no effect of context reinstatement on source memory. In the continuous recognition tasks employed by Craik and Kirsner (1974), there was no effect of voice reinstatement on accuracy in judging whether a word was previously heard in a male or female voice. Similarly, Kirsner (1973) found no effect of font case reinstatement on the ability to determine whether a word was previously seen in an uppercase or lowercase font (Exp. 1), although a positive cuing effect did arise when nonword letter strings were used as stimuli (Exp. 2). Work by the present authors showed that reinstating the font size of a word did not improve source memory for font size, and reinstating the location of a word did not improve source memory for location (Starns \& Hicks, 2005). Vogt and Bröder (2007) replicated the null effects for font size and location, and they also found that reinstating text color or pictures of background scenes did not impact source memory for color or background information, respectively. Moreover, attempts to cue memory for one source dimension by reinstating information along another dimension have failed (Starns \& Hicks, 2005, 2008; Vogt \& Bröder, 2007). In addition to these published failures to obtain cuing effects, a number of unsuccessful cuing studies might remain unpublished due to the difficulty of publishing null results.

Some researchers have reported more accurate source memory when source details were reinstated at retrieval (Dodson \& Shimamura, 2000; Leynes et al., 2003; Palmeri et al., 1993; Roediger et al., 2004; Vogt \& Bröder, 2007), but the potential memory effects are obscured by response biases. For example, Dodson and Shimamura tested recognition memory and source memory for male and female voices across a variety of conditions including matched-voice cues, mismatched-voice cues, and no cues. Relative to the uncued condition, participants produced more claims of "male" when a male cue was presented at test and more claims of "female" when a female cue was presented at test. This bias mimics memory effects, because it results in more correct source claims when the study and test sources match than when they mismatch.

To disentangle memory and bias effects, Dodson and Shimamura (2000) fit their data using multinomial processing tree models (Batchelder \& Riefer, 1990; Bayen, Murnane, \& Erdfelder, 1996). Multinomial models propose hypothetical cognitive states that underlie performance on a joint recognition and source memory test, and each parameter estimates the probability of experiencing a discrete cognitive state. Panel A of Fig. 1 shows how internal states map to overt responses in a standard multinomial model for items that were studied in Source A (Bayen et al., 1996). The $D$ parameter estimates the probability of detecting that the item was studied. When item detection fails, participants must make an uninformed guess as to whether or not the item was on the study list, and they guess "old" (studied) with probability $b$ or "new" (not studied) with probability $1-b$. The $d$ parameter estimates the probability of correctly detecting the source of a studied item. When item detection succeeds but source detection fails, participants guess that the item was studied in Source A with probability $a$. For items called "studied" on the basis of a guess, source detection never succeeds, and participants guess "Source A" with probability $g$. The model for Source B items is identical to the one displayed, except that successful source detection $(d)$ leads to a "Source B" response. For new items, successful item detection leads to a "new" response.

The model results from Dodson and Shimamura (2000) indicated that source reinstatement improved source memory in addition to introducing response biases. Similarly, multinomial modeling by Vogt and Bröder (2007) showed that reinstating font
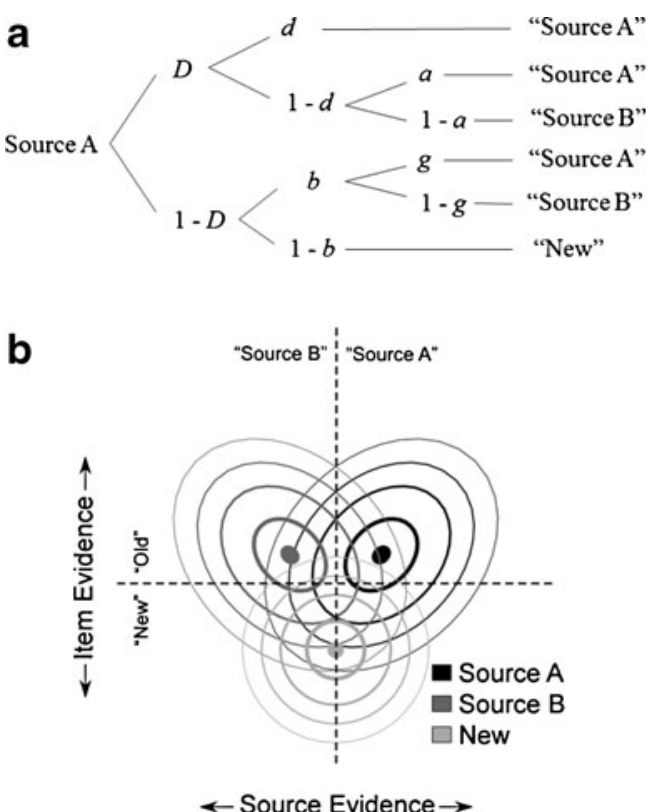

Fig. 1 Multinomial processing tree model (panel A) and bivariate signal detection model (panel B) for recognition and source judgments. See the text for model descriptions 
type (print vs. script) affected source memory for font type (in the same experiments in which they found null memory effects for font size, location, color, and background pictures).

\section{Null cuing effects and internal reinstatement}

Collectively, existing source cuing studies demonstrate that source reinstatement effects are surprisingly fragile. Some theorists have explained these null effects by proposing that participants internally reinstate cues when external cues are unavailable at retrieval (Bjork \& Richardson-Klavehn, 1989; Murnane \& Phelps, 1994; Smith, 1979). That is, external cues may be ineffective because the information they provide is redundant with information internally reinstated by participants on uncued trials. We will call this explanation of null context effects the internal-cuing account.

A study by Smith (1979) attempted to manipulate the effectiveness of internal reinstatement by varying the number of contexts that competed for retrieval with the studyroom context. On the first day of the experiment, participants performed five tasks, one of which was learning a word list. For one group of participants, the word list was learned in one room and all other tasks were performed in a single different room (two-context group). For another group of participants, the word list was learned in one room and the remaining four tasks were performed in four different rooms (five-context group). On the second day of the experiment, half of the participants in each group performed free recall in the learning room, and half performed free recall in a different room with instructions to try to remember the learning room to help them recall words. Results showed no significant difference between same- and different-context participants in the two-context condition, but an advantage for same- over different-context participants in the five-context condition. With only two study contexts, it was easy for participants to remember the unique details of the encoding room, providing them the opportunity to internally reinstate context information. Increasing the number of contexts encountered on the first day made it difficult for participants to specifically remember the encoding room; therefore, internal reinstatement could not compensate for the lack of external cues.

Smith's (1979) results demonstrate that internal reinstatement can eliminate external cuing effects in item recall, and we sought to extend these results to source memory. Notably, internal reinstatement could be easily achieved by participants in standard source tasks. Usually, participants encounter only two sources that they experience many times throughout the encoding phase, so they should be intimately familiar with the source characteristics that they need to internally reinstate at test. Thus, evidence that source cues affect discrimination might be equivocal because external source cues are redundant with the internal cues generated by participants.

\section{Separating memory from bias}

In previous source cuing studies, researchers have used multinomial decision models to determine whether cues affect source memory in addition to decision biases (Dodson \& Shimamura, 2000; Vogt \& Bröder, 2007). Importantly, alternative decision models make different predictions for the relative impact of bias on correct and incorrect responses, and this difference in assumptions can lead to different conclusions. For example, Dube, Rotello, and Heit (2010) demonstrated that multinomial models and continuous signal detection models produce different interpretations of belief-bias effects.

For joint recognition and source judgments, the main competitor for multinomial models is the bivariate signal detection model shown in Fig. 1, panel B (Banks, 2000; DeCarlo, 2003). For each type of item, memory evidence varies continuously on both item and source dimensions. The figure shows a bivariate Gaussian distribution for each item type. The ovals are equal-density contours, with thicker lines representing higher probability densities. The Source A and Source B distributions have higher average item strengths than new items, and Source A items tend to have higher values than Source B items on the source dimension. Source memory can be measured in terms of $d^{\prime}$, the distance between the means of the two source distributions measured in units of their common standard deviation. For the studied items, the source distributions diverge with increasing item strength; that is, items that support strong recognition memory also tend to produce accurate source memory. The horizontal line is the recognition criterion dividing "new" and "old" responses, and the vertical line is the source criterion dividing "Source A" and "Source B" responses. Bias effects are modeled in terms of the position of the response criteria, and memory effects are modeled in terms of the evidence distributions.

The model in Fig. 1B clearly demonstrates another impact of bias on cuing results: Source discrimination for recognized items depends on the bias to say "old" for the recognition decision (DeCarlo, 2003). When people are conservative, only the items with strong item evidence are called "old" and assigned to a source, and these items also tend to have highly discriminative source evidence. Therefore, conservative recognition responding leads to better source performance than does liberal responding. Notably, providing a context cue from the study phase can lead to more liberal recognition responding (e.g., Murnane \& Phelps, 1993), which would decrease source performance in the cued condition and potentially counteract any boost in source memory provided by the cues. This relationship between recognition bias and source performance also holds for multinomial models, because more liberal recognition responding reflects a greater willingness to guess "old" when item detection fails, and all source responses are guesses in this circumstance (Fig. 1A). 
The proponents of continuous versus multinomial models are currently engaged in a lively debate regarding which one best describes the nature of memory evidence and decision processes (Bröder \& Meiser, 2007; Dube \& Rotello, 2012; Dube, Starns, Rotello, \& Ratcliff, 2012; Erdfelder \& Buchner, 1998; Klauer \& Kellen, 2010; Malmberg, 2002; Schütz \& Bröder, 2011; Starns, Hicks, Brown, \& Martin, 2008; Wixted, 2007). For our purposes, the important point is that the debate is unresolved, so conclusions about memory processes should not rest on the validity of one particular model.

\section{The present experiments}

Our primary goal was to test the internal-cuing account by influencing the effectiveness of internal reinstatement. Participants learned words that appeared with either male or female faces. Separate groups of participants encountered either a single male and female face or multiple male and female faces in the encoding phase. Our predictions followed the logic of the Smith (1979) experiment: When participants study multiple faces for each gender, they should not be able to internally reinstate all of the studied faces on each test trial. In contrast, with only one face for each gender, internally reinstating the studied faces should be relatively easy. Thus, the internal-cuing account predicts that source cues should have a larger effect on memory performance in the multiple-face than in the singleface condition.

In Experiment 2, we used a retrieval-based manipulation to test the internal-cuing account. Using the multiple-face encoding list, we compared a standard test condition to a test that was structured to facilitate internal reinstatement. Specifically, the test was organized into blocks, and participants were informed that all items within each block were studied with one of two faces (one male and one female). The internal-cuing account predicts that even participants who study multiple faces should show no cuing effects if internal reinstatement is made possible at test.

A secondary goal was to develop a cuing procedure that has a minimal impact on source response bias. To achieve this, we presented information from both sources on the cued trials; that is, participants saw both a male and a female face. With this procedure, cues should not strongly bias responses in the direction of a single source, such as a male cue producing more "male" responses even for items studied in the female source.

To address the effect of recognition bias on source performance, we had participants provide source decisions for every studied item. In terms of the bivariate model shown in Fig. 1B, this allowed us to estimate the distance between the male and female distributions on the source dimension without the confounds produced by selection effects (DeCarlo, 2003). We achieved this by simply calculating $d^{\prime}$ for each participant on the basis of overall responding for male and female items. In Experiments 1 and 2, participants made a male/female decision for each test item without making recognition judgments. In Experiments $3 \mathrm{a}$ and $3 \mathrm{~b}$, participants made recognition decisions and were required to specify the source for all target items, regardless of whether they had responded "old" or "new."

We also addressed response bias by confirming that our conclusions were supported by both a signal detection model and a multinomial model. In addition to $d^{\prime}$, we evaluated our results in terms of hit rates minus false-alarm rates, the measure appropriate for a multinomial model with equal detection probabilities across item types. Moreover, we applied a multinomial model to the recognition and source results from Experiments $3 \mathrm{a}$ and $3 \mathrm{~b}$.

\section{Experiment 1}

Participants studied words that were paired with either one male and one female face (single-face condition) or one of eight male and eight female faces (multiple-face condition). All participants completed a source test for gender. On cued trials, the face studied with the test word was displayed at test along with a face from the other gender. On uncued trials, only the word was displayed. Participants should be able to internally reinstate the male and female face in the single-face condition; therefore, the internal-cuing account predicts little or no cuing effect in this condition. The multiple-face condition should create internal reinstatement difficulties; therefore, the external cues should improve source memory.

\section{Method}

Participants Data were collected from 92 Louisiana State University undergraduates who received course extra credit for volunteering. Participants were randomly assigned to the single-face and multiple-face conditions in equal numbers.

Design This experiment followed to 2 (face number) $\times 2$ (cuing condition) design. Face number was manipulated between subjects, and cuing condition was manipulated within subjects.

Materials A total of 96 words were selected from the MRC Psycholinguistic Database (www.psy.uwa.edu.au/mrcdatabase/ uwa mrc.htm). All words were between five and eight letters in length, had familiarity ratings between 500 and 700, and had concreteness ratings between 300 and 500. Eight male and eight female faces were selected from Maner et al. (2003). The encoding list contained 96 words, with 48 words seen with a female face and 48 seen with a male face. The assignment of gender to individual words was counterbalanced across participants. For the single-face group, one of the male and one of the 
female faces was selected at random for each participant, and these two faces appeared throughout the study phase. In the multiple-face group, each of the eight male and eight female faces was randomly paired with six words. The test list contained all 96 studied words, with half of the words studied in each gender appearing with no face at test, and half appearing with face cues at test. On cued trials, the test word appeared near the top of the computer screen. Immediately below the word, a picture of a male face and a picture of a female face appeared side-by-side, with the female face on the left and the male face on the right. One face was always the individual face paired with the test item in the study phase. For the gendermismatched face, the alternative face was presented in the single-face condition, or an individual face was selected at random in the multiple-face condition. All faces appeared an equal number of times on the test.

Procedure Before the study phase, participants were told that they would see words paired with faces, and that their memory would be tested later in the experiment. They were asked to imagine the person that they saw on each trial "demonstrating" the word they saw. For example, participants were told that, if the word was a verb, they could imagine the person in the picture acting out the word. Conversely, if the word was a noun, they could imagine the person in the picture holding up or pointing to the word's referent. After the instructions, the word-face pairs on the study list appeared in random order, and each remained on the screen for $5 \mathrm{~s}$ with $200 \mathrm{~ms}$ of blank screen between presentations. The pictures appeared in the center of the computer screen, and words appeared below the picture. Next, participants performed a distracter task for $3 \mathrm{~min}$ in which they rearranged the segments of a scrambled picture on the computer screen. None of the pictures in the distracter task depicted human faces. The test instructions informed participants that they would see words that appeared in the study phase, and that they must decide whether each word appeared with a male or a female face. They entered a confidence rating on a 6-point scale using the numbers 1-6 on the keyboard. The points of the rating scale were labeled "Very Sure Female," "Sure Female," "Guess Female," "Guess Male," "Sure Male," and "Very Sure Male." Participants were told that they would see a male and a female face on some of the test trials, and that one of the faces would always be the face studied with the test word.

\section{Results and discussion}

We assessed source discrimination by calculating $d^{\prime}$ scores with the standard formula $\left[d^{\prime}=z(\mathrm{HR})-z\right.$ (FAR) $]$, with the hit rate defined as the proportion of male items called "male" (with any level of confidence) and the false-alarm rate defined as the proportion of female items called "male." This measure assumes that an equal-variance signal detection model underlies source performance (equivalent to the source dimension in Fig. 1B), and this model has been successful in accommodating source data (e.g., Slotnick \& Dodson, 2005). We used the Snodgrass and Corwin (1988) correction to avoid undefined $d^{\prime}$ values resulting from hit rates equal to one and false alarm rates equal to zero (i.e., a constant of .5 was added to the frequency of "male" and "female" responses before calculating the hit and false-alarm rates). Results for hit rate minus false-alarm rate matched those for the $d^{\prime}$ measure in all cases except one, so we report only the $d^{\prime}$ analyses and note the one discrepancy (in Exp. 3).

Table 1 shows the source data for Experiment 1. For the multiple-face group, providing cues resulted in more "male" responses to male items and fewer "male" responses to female items, and the $d^{\prime}$ 'scores indicated better source memory on cued versus uncued trials. A 2 (face number) $\times 2$ (cuing condition) analysis of variance (ANOVA) on source discrimination $\left(d^{\prime}\right)$ produced a main effect of face number, $F(1,90)=4.87, p<.05$. Source performance was lower in the multiple-face condition $(M=.93)$ than in the single-face condition $(M=1.28)$. The ANOVA also revealed a main effect of cue, $F(1,90)=5.31$, $p<.05$, and more critically, a significant interaction, $F(1,90)=7.99, p<.01$. Specific contrasts showed that cues significantly affected source memory in the multiple-face condition, $t(45)=3.57, p<.01$, but not in the single-face condition, $t(45)=0.38, p=.71$. The standardized effect size (Cohen's $d$ ) was 0.53 in the multiple-face condition, indicating a medium-sized effect by Cohen's (1988) guidelines, as compared to 0.06 in the single-face condition, indicating a very

Table 1 Source memory performance in Experiment 1

\begin{tabular}{|c|c|c|c|c|c|c|}
\hline \multirow[t]{3}{*}{ Cuing Condition } & \multicolumn{6}{|c|}{ Face Number and Source Measure } \\
\hline & \multicolumn{3}{|c|}{ Multiple Face } & \multicolumn{3}{|c|}{ Single Face } \\
\hline & " $M " \mid M$ & “ $M " \mid \mathrm{F}$ & $d^{\prime}$ & “M" $\mid M$ & “ $M " \mid \mathrm{F}$ & $d^{\prime}$ \\
\hline Uncued & $.66(.02)$ & $.38(.02)$ & $0.76(0.10)$ & $.73(.02)$ & $.28(.03)$ & $1.29(0.15)$ \\
\hline Cued & $.71(.02)$ & $.31(.02)$ & $1.11(0.11)$ & $.73(.02)$ & $.29(.02)$ & $1.26(0.13)$ \\
\hline
\end{tabular}

" $M$ " $\mid M$ is the proportion of male items called "male"; " $M " \mid F$ is the proportion of female items called "male." Standard errors are in parentheses 
small (if any) effect. Thus, the results showed clear evidence for the interaction predicted by the internal-cuing account.

\section{Experiment 2}

Our explanation for the difference between the single-face and multiple-face conditions focuses on processes operating at retrieval. That is, we propose that studying multiple faces disrupts internal reinstatement abilities, which impairs source performance unless an appropriate external cue is provided. However, the actual manipulation was achieved at encoding. It is possible that uncued performance was worse for the multiple-face condition because having more faces at encoding impaired learning (similar to the speaker-variability effect; e.g., Martin, Mullennix, Pisoni, \& Summers, 1989). Moreover, some difference in initial learning could have also rendered the multiple-face condition more susceptible to the cuing manipulations. For example, each individual face was paired with 48 words in the single-face condition versus six words in the multiple-face condition, perhaps rendering the former ineffective due to cue overload (Watkins \& Watkins, 1975). Our account would be most strongly supported by a retrieval manipulation that impacts internal reinstatement and interacts with the effects of external cues. We designed Experiment 2 to meet this goal.

All participants studied multiple male and female faces and completed a test in which they saw either no faces or two face cues as in the previous experiment. One group of participants took a standard test with words from different faces randomly intermixed. Internal reinstatement should be ineffective in this condition, and we should see better discrimination on cued trials. Another group of participants completed a test that was organized into blocks. Each block of the test contained items that were studied with one of the male faces and one of the female faces. Participants were shown pictures of the two relevant faces before each block of the test. As a result, participants knew exactly which faces needed to be internally reinstated to guide performance. Given the ease of internal reinstatement induced by this preblock exposure, discriminability should be high in both the cued and uncued conditions.

\section{Method}

Participants The blocked and random conditions each included 30 participants from Louisiana State University.

Design This experiment conformed to a 2 (test composition) $\times 2$ (cuing condition) design. Test composition was manipulated between subjects, and cuing condition was manipulated within subjects.

Materials and procedure All methodological details of the random-test condition matched those of the multiple-face condition in Experiment 1. For the blocked test condition, the test instructions additionally informed participants that the test items would be grouped in small blocks, and that each block contained items studied with one male and one female face. During the test, each block was introduced by showing the male and female face for that block. Each block contained with six cued and six uncued trials. The test comprised eight blocks.

\section{Results and discussion}

Table 2 shows the source memory measures for this experiment. The $d^{\prime}$ scores show a strong cuing effect in the random test group, which replicates results from the multiple-face conditions in Experiment 1. Once again, cues improved performance for items from both sources: There were more "male" responses for male items and fewer "male" responses for female items. In contrast, uncued performance was nearly as high as cued performance in the blocked test group. A 2 (cue) $\times 2$ (test composition) ANOVA revealed no difference between the random and blocked groups, $F(1,58)=1.50$, $p=.226$, but did reveal a significant cuing effect, $F(1,58)=$ $17.26, p<.001$, that was qualified by a significant interaction, $F(1,58)=6.30, p<.05$. The interaction arose because cues significantly improved performance in the random test group, $t(29)=4.83, p<.001$, but did not impact performance in the blocked test group, $t(29)=1.14, p=.265$. The standardized effect size for the cuing effect was .88 in the random condition and .21 in the blocked condition.

Table 2 Source performance in Experiment 2

\begin{tabular}{|c|c|c|c|c|c|c|}
\hline \multirow[t]{3}{*}{ Cuing Condition } & \multicolumn{6}{|c|}{ Test Composition and Source Measure } \\
\hline & \multicolumn{3}{|l|}{ Random } & \multicolumn{3}{|l|}{ Blocked } \\
\hline & " $M " \mid M$ & “ $M " \mid \mathrm{F}$ & $d^{\prime}$ & “M" $\mid M$ & “M”| F & $d^{\prime}$ \\
\hline Uncued & $.65(.03)$ & $.36(.02)$ & $0.75(0.12)$ & $.73(.02)$ & $.30(.02)$ & $1.13(0.09)$ \\
\hline Cued & $.75(.02)$ & $.30(.03)$ & $1.26(0.15)$ & $.72(.03)$ & $.26(.02)$ & $1.26(0.13)$ \\
\hline
\end{tabular}

" $M$ " $\mid M$ is the proportion of male items called "male"; " $M " \mid \mathrm{F}$ is the proportion of female items called "male." Standard errors are in parentheses 
The pattern across the test composition variable in this experiment is very similar to the pattern observed for the number-of-faces variable in Experiment 1 . In the blocked group, participants discriminated sources as effectively on uncued trials as on cued trials (at least in a statistical sense). In contrast, uncued performance was relatively poor in the random group, and presenting cues brought performance back to the level observed in the blocked test group. This pattern provides strong evidence for the internal-cuing account. Participants in the blocked test group know exactly which male and female faces they should internally reinstate on uncued trials; thus, appropriate source cues are available even when they are not externally presented. In the random test group, participants cannot predict which specific faces should be internally reinstated on uncued trials. This leads to poor performance in the uncued condition, but the deficit is eliminated when external cues are provided to focus memory assessments. Critically, the different results in the random and blocked conditions must be attributed to retrieval factors, because the two groups encountered identical encoding procedures.

In hindsight, the lack of a cuing effect in the blocked condition seems unsurprising: The procedures allowed participants to easily turn any uncued trial into a cued trial by imagining the male or female face for the block. However, the same is true for the standard procedures in source cuing experiments (Craik \& Kirsner, 1974; Dodson \& Shimamura, 2000; Kirsner, 1973; Leynes et al., 2003; Roediger et al., 2004; Starns \& Hicks, 2005; Vogt \& Bröder, 2007). In these experiments, participants study only two sources that appear many times in the study list, and they complete the test shortly thereafter. So participants should have detailed memory for the characteristics of each source to internally reinstate, and mixing cued trials into the test provides frequent reminders of the source characteristics. Just like our participants in the blocked condition, those in a standard source design should have no trouble making nominally uncued trials into cued trials. In this light, it is no surprise that null results have been common in the source cuing literature, and that we observed no appreciable cuing in the single-face condition of Experiment 1.

\section{Experiments $3 \mathrm{a}$ and $3 \mathrm{~b}$}

According to the bivariate signal detection model in Fig. 1, our cuing effects in Experiments 1 and 2 can be attributed to differences in source memory. Providing a cue increased both "male" responses for male items and "female" responses for female items, a pattern that can only be accommodated by decreasing the degree of overlap between the male and female distributions on the source dimension (either by moving the means farther apart or decreasing the variance). Moreover, participants made source judgments for every studied item, so the source results cannot be distorted by selection effects resulting from changing the recognition criterion (DeCarlo, 2003).

The picture is more complex when one interprets our results using a multinomial decision model. Given that cues increased accuracy for items from both sources, our results still cannot be explained in terms of guessing biases alone. ${ }^{1}$ Instead, the results indicate that a higher proportion of male items were detected as male and a higher proportion of female items were detected as female. However, this result can emerge in two different ways in the multinomial framework. Standard multinomial models have no decision paths that combine the internal states of failed item detection $(1-D)$ and accurate source detection $(d)$; instead, failed item detection is always associated with source guessing (see Fig. 1A; Bayen et al., 1996). Thus, source cues could either increase the probability of source detection for items that were successfully detected as "old," or increase the proportion of items detected as "old" without affecting the probability of source detection for these items. Murnane and Bayen (1996) also noted that measures of source memory are often confounded by the level of item memory using a general theoretical framework as opposed to a particular decision model (although they did not consider cases in which source claims were made for all items, as in our first two experiments).

To determine whether cues affect source detection, Experiments $3 \mathrm{a}$ and $3 \mathrm{~b}$ included nonstudied items on the test and required recognition judgments prior to the source decisions. Our primary goal was to determine whether multinomial modeling supports our claim that cues enhance source memory in the multiple-face condition but not in the singleface condition. To address this issue, we compared the relative fit of models in which source memory could or could not vary across cued and uncued trials. We predict that equating source memory should result in a significantly worse fit in the multiple-face condition but not the single-face condition.

Our primary interest was whether or not cues affect source memory, but we also tested for cuing effects on recognition memory. Cuing effects on recognition bias were of particular interest, because they can impact source judgments via selection effects (DeCarlo, 2003). As we noted in the introduction, becoming more biased to respond "old" produces a decrease in source accuracy for items called "old" in the bivariate signal detection approach (DeCarlo, 2003), as well as in the multinomial models that have been applied to recognition and source data (e.g., Bayen et al., 1996). Moreover, empirical results

\footnotetext{
${ }^{1}$ Technically, a variable that only affects bias in a multinomial model can increase performance for both sources, under these conditions: (1) One source has higher item memory that the other, (2) the variable produces a bias toward the stronger source when item detection succeeds, and (3) the variable produces a bias against the stronger source when item detection fails. However, Experiments $3 \mathrm{a}$ and $3 \mathrm{~b}$ showed that item memory was very similar across our male and female sources. In this circumstance, a mere bias effect cannot increase accuracy for both sources.
} 
have demonstrated that source memory is better for words with stronger item memories (e.g., Slotnick \& Dodson, 2005), so one need not assume a particular decision model to predict that source memory will suffer if weaker items are considered "old" (i.e., if responding becomes more liberal). If cues promote a bias to say "old," this could potentially counteract any benefit to source memory and eliminate cuing effects for source performance conditionalized on an "old" response. To evaluate these possible selection effects, we required source judgments for all studied words. That is, participants made source decisions even when they incorrectly called a studied word "new." Requiring source responses for all studied items will allow us to determine whether results replicated those of Experiment 1, and will also allow us to calculate source $d^{\prime}$ without the distortions produced by selection effects.

We ran Experiment $3 b$ to ensure that cuing effects were not eliminated when performance levels were high. This experiment was identical to the multiple-face condition in Experiment $3 \mathrm{a}$, except that the studied items were presented twice. If strong learning eliminates cuing effects, then the cuing results from Experiment 1 should replicate in those of Experiment 3a but not Experiment 3b.

\section{Method}

Participants Experiment 3a included 34 participants in the single-face group and 30 in the multiple-face group. Of these
64 participants, 43 were from the subject pool at Louisiana State University, and 21 were from the subject pool at the University of Massachusetts Amherst. Participants from both pools were randomly assigned to either the single-face or multiple-face conditions. Experiment $3 \mathrm{~b}$ included 24 participants from LSU.

Design The design of Experiment 3a was identical to Experiment 1. Experiment $3 \mathrm{~b}$ only included the multiple-face condition.

Materials and procedure For Experiment 3a, all methodological details matched Experiment 1, with the following exceptions: (1) 48 new items were added to the test, with word sets counterbalanced between the male source, female source, and new items across participants; (2) half of the new items appeared with face cues at test (with each face used an equal number of times across trials in the multipleface condition), and the other half had no faces at test; (3) participants were first asked to make an "old"/"new" response by pressing the "z" or "/" keys, respectively, and then they used the same keys to make a "male"/"female" response for all studied words (regardless of the recognition response) and for any new item that they called "old"; and (4) the puzzle task between the study and test lists was eliminated. Experiment $3 \mathrm{~b}$ only used the multiple-face condition and participants saw the entire study list twice in a new random order each time.

Table 3 Recognition performance in Experiments $3 \mathrm{a}$ and $3 \mathrm{~b}$

\begin{tabular}{|c|c|c|c|c|c|c|}
\hline \multirow[t]{3}{*}{ Cuing Condition and Gender } & \multicolumn{6}{|c|}{ Face Number and Recognition Measure } \\
\hline & \multicolumn{3}{|c|}{ Multiple Face } & \multicolumn{3}{|c|}{ Single Face } \\
\hline & HR & FAR & $d_{\mathrm{UV}}$ & HR & FAR & $d_{\mathrm{UV}}$ \\
\hline \multicolumn{7}{|l|}{ Experiment $3 \mathrm{a}$} \\
\hline \multicolumn{7}{|l|}{ Uncued } \\
\hline Male & $.66(.03)$ & $.14(.02)$ & $1.69(0.15)$ & $.75(.02)$ & $.16(.02)$ & $1.94(0.14)$ \\
\hline Female & $.68(.02)$ & $.14(.02)$ & $1.75(0.13)$ & $.76(.02)$ & $.16(.02)$ & $1.99(0.15)$ \\
\hline \multicolumn{7}{|l|}{ Cued } \\
\hline Male & $.77(.02)$ & $.21(.03)$ & $1.80(0.13)$ & $.83(.02)$ & $.17(.03)$ & $2.28(0.17)$ \\
\hline Female & $.78(.02)$ & $.21(.03)$ & $1.87(0.12)$ & $.80(.02)$ & $.17(.03)$ & $2.11(0.15)$ \\
\hline \multicolumn{7}{|l|}{ Experiment $3 b$} \\
\hline \multicolumn{7}{|l|}{ Uncued } \\
\hline Male & $.76(.03)$ & $.11(.02)$ & $2.27(0.19)$ & & & \\
\hline Female & $.75(.03)$ & $.11(.02)$ & $2.19(0.18)$ & & & \\
\hline \multicolumn{7}{|l|}{ Cued } \\
\hline Male & $.84(.02)$ & $.22(.04)$ & $2.25(0.18)$ & & & \\
\hline Female & $.87(.02)$ & $.22(.04)$ & $2.37(0.23)$ & & & \\
\hline
\end{tabular}

The same false-alarm rate values are reported in the "Male" and "Female" rows. HR = hit rate; FAR = false-alarm rate. $d_{\mathrm{UV}}$ is the distance between the means of the target and lure evidence distributions in a signal detection model in which the target evidence is $25 \%$ more variable than the lure evidence. Standard errors are in parentheses 
Table 4 Overall source performance for Experiments $3 \mathrm{a}$ and $3 \mathrm{~b}$

\begin{tabular}{|c|c|c|c|c|c|c|}
\hline \multirow[t]{3}{*}{ Cuing Condition } & \multicolumn{6}{|c|}{ Face Number and Source Measure } \\
\hline & \multicolumn{3}{|c|}{ Multiple Face } & \multicolumn{3}{|c|}{ Single Face } \\
\hline & " $M " \mid M$ & $" M " \mid \mathrm{F}$ & $d^{\prime}$ & “ $M " \mid M$ & “ $M " \mid \mathrm{F}$ & $d^{\prime}$ \\
\hline \multicolumn{7}{|l|}{ Experiment 3a } \\
\hline Uncued & $.71(.02)$ & $.32(.03)$ & $1.09(0.13)$ & $.75(.02)$ & $.25(.02)$ & $1.43(0.13)$ \\
\hline Cued & $.74(.02)$ & $.23(.02)$ & $1.39(0.10)$ & $.77(.02)$ & $.25(.03)$ & $1.50(0.14)$ \\
\hline \multicolumn{7}{|l|}{ Experiment $3 b$} \\
\hline Uncued & $.74(.03)$ & $.27(.03)$ & $1.41(0.21)$ & & & \\
\hline Cued & $.77(.03)$ & $.19(.03)$ & $1.79(0.21)$ & & & \\
\hline
\end{tabular}

" $M$ " $\mid M$ is the proportion of male items called "male"; " $M$ " $\mid \mathrm{F}$ is the proportion of female items called "male." Standard errors are in parentheses

\section{Results and discussion}

Recognition memory The recognition data appear in Table 3. For the multiple-face condition, participants made more "old" responses for cued than for uncued trials, resulting in an increase in both hit rate and false-alarm rate. This effect on response bias was evident in both Experiments $3 a$ and $3 b$. The single-face participants in Experiment 3a did not show clear evidence of this bias: Hit rates increased slightly when cues were provided, but false-alarm rates were very similar with and without cues.

A 2 (cue) $\times 2$ (face number) $\times 2$ (gender) ANOVA on hit rates from Experiment 3 a showed a main effect of face number, $F(1,62)=6.63, p<.05$. Hit rates were higher in the single-face (.79) than in the multiple-face (.72) condition. Hit rates were also higher for cued trials (.79) than for uncued trials (.71), $F(1,62)=60.48, p<.001$. The cuing effect was larger for multiple-face participants (.11) than for single-face participants (.06), which produced a significant interaction, $F(1,62)=5.10$, $p<.05$. A 2 (cue) $\times 2$ (gender) ANOVA on the Experiment $3 \mathrm{~b}$ data showed only an effect of cuing condition, $F(1,23)=16.73$, $p<.001$. Hit rates were again higher for cued (.85) than for uncued (.76) trials.

For the false-alarm rate data from Experiment 3a, a 2 (cue) $\times 2$ (face number) ANOVA showed a main effect of cuing condition, $F(1,62)=9.81, p<.01$, that was qualified by an interaction, $F(1,62)=4.07, p<.05$. The single-face participants showed no evidence of a cuing effect, $t(33)=0.80, p=.429$. In contrast, the multiple-face participants showed an increase in false-alarm rate from uncued (.14) to cued (.21) trials, $t(29)=$ $3.60, p=.001$. This effect was replicated in Experiment $3 \mathrm{~b}$ (uncued $=.11$, cued $=.22), t(23)=3.56, p<.01$.

Our next analyses focused on whether cues improved recognition memory in addition to promoting a bias to respond "old." We did not use $d$, , because target evidence is more variable than lure evidence in recognition memory (e.g., Ratcliff, Sheu, \& Gronlund, 1992). Instead, we calculated an analogous measure based on the assumption that targets are $25 \%$ more variable than lures, a value that is consistent with empirical results (see Wixted, 2007, for a review). We call this measure $d_{\mathrm{UV}}$ (where UV stands for "unequal variance"). As with the source analyses, we also

Table 5 Source performance for items called "old" in Experiments 3a and 3b

\begin{tabular}{|c|c|c|c|c|c|c|}
\hline \multirow[t]{3}{*}{ Cuing Condition } & \multicolumn{6}{|c|}{ Face Number and Source Measure } \\
\hline & \multicolumn{3}{|c|}{ Multiple Face } & \multicolumn{3}{|c|}{ Single Face } \\
\hline & " $M " \mid M$ & “M” $\mid \mathrm{F}$ & $d^{\prime}$ & “ $M " \mid M$ & “M" $\mid \mathrm{F}$ & $d^{\prime}$ \\
\hline \multicolumn{7}{|l|}{ Experiment $3 \mathrm{a}$} \\
\hline Uncued & $.80(.03)$ & $.26(.03)$ & $1.53(0.14)$ & $.81(.03)$ & $.18(.02)$ & $1.89(0.15)$ \\
\hline Cued & $.78(.02)$ & $.20(.02)$ & $1.63(0.12)$ & $.82(.02)$ & $.19(.02)$ & $1.88(0.15)$ \\
\hline \multicolumn{7}{|l|}{ Experiment $3 b$} \\
\hline Uncued & $.81(.03)$ & $.21(.03)$ & $1.84(0.21)$ & & & \\
\hline Cued & $.79(.04)$ & $.18(.03)$ & $1.97(0.25)$ & & & \\
\hline
\end{tabular}

" $M$ " $\mid M$ is the proportion of male items called "male"; " $M " \mid F$ is the proportion of female items called "male." Standard errors are in parentheses 
analyzed corrected performance (hit rate minus false-alarm rate).

For Experiment $3 \mathrm{a}$, a 2 (cue) $\times 2$ (face number) $\times 2$ (gender) ANOVA on $d_{\mathrm{UV}}$ revealed only a significant effect of cue, $F(1,62)=6.21, p=.015$. Recognition performance was better for cued (2.02) than for uncued trials (1.84). However, this effect did not replicate in Experiment 3b, which showed similar levels of performance for cued (2.31) and uncued (2.23) trials, $F(1,23)=0.42, p=.521$.

Overall source memory We first consider source memory across all studied items (regardless of whether they were called "old" or "new" for the recognition decision). These data appear in Table 4. The multiple-face results replicated the pattern seen in Experiment 1: Providing a cue improved accuracy for both male and female items, resulting in an increase in source discriminability. Also replicating Experiment 1 , the single-face group showed little evidence that cues improved source memory.

For Experiment 3a, a 2 (cue) $\times 2$ (face number) ANOVA on source $d^{\prime}$ showed a significant effect of cue, $F(1,62)=$ $7.95, p<.01$, with no effect of face number, $F(1,62)=1.86$, $p=.177$. The single-face group showed no strong evidence of a cuing effect, $t(33)=0.83, p=.411$, and the standardized effect size was below guidelines for a small effect $(d=0.14$; Cohen, 1988). In contrast, the multiple-face group had higher performance for cued than for uncued trials, $t(29)=2.86$, $p<.01, d=0.52$. The interaction between cue and face number approached convention levels of significance, $F(1,62)=3.25, p=.076$. This analysis was the one point of disagreement between $d^{\prime}$ and corrected source performance (hits minus false alarms), as the interaction did reach significance for the latter measure, $F(1,62)=5.30, M S E=.013, p<$ .05 . Experiment $3 \mathrm{~b}$ also demonstrated a positive cuing effect with multiple faces, $t(23)=3.83, p=.001, d=0.78$.

Conditionalized source memory Here, we evaluate source performance for targets that were correctly called "old," which is shown in Table 5. Notably, the cuing effects evident in the overall source data are substantially reduced in the conditionalized data. This is not surprising, given that conditionalized source performance should decrease when recognition responding is more liberal, and participants were more liberal with cues than without. Analyses on conditionalized source $d^{\prime}$ showed no significant effects for any of the variables in either Experiment $3 a$ or $3 b$ (lowest $p$ value $=.108$, for the face number effect in Exp. 3a).

Multinomial modeling We applied a standard multinomial model to ensure that our results reflect an effect of cues on source memory (e.g., Bayen et al., 1996). The Appendix describes our fitting procedures in detail, and Table 6 reports the parameter values and fit statistics. The analyses of primary interest concerned the effect of cues on the source detection parameter $\left(d_{\mathrm{C}}\right.$ vs. $d_{\mathrm{NC}}$ in Table 6$)$. For the single-face participants in Experiment 3a, source memory was very similar on cued (.81) and uncued (.81) trials, and equating source memory across the cuing conditions did not significantly worsen the fits, $G^{2}(1)=0.001, p=.975$. In contrast, the multiple-face participants in Experiment 3a showed higher source memory for cued trials (.81) than for uncued trials (.68), and the same pattern emerged in Experiment $3 \mathrm{~b}$ (.83 vs. .72). In both cases, equating source memory across the cuing conditions produced a significant change in fit [Exp. 3a: $G^{2}(1)=5.262, p<.05$; Exp. 3b: $\left.G^{2}(1)=4.531, p<.05\right]$. Thus, the multinomial model supports our central claim: Source cues improve source memory in the multiple-face condition but not the single-face condition.

We also used the models to evaluate the effect of cues on item memory. The multinomial analyses confirmed that cues affected recognition bias, with more liberal responding on cued than on uncued trials [Exp. 3a: single-face, $G^{2}(1)=8.142$, $p<.005$; multiple-face, $G^{2}(1)=39.004, p<.001$; Exp. 3b: $\left.G^{2}(1)=53.015, p<.001\right]$. Although cues appear to have conferred a small item detection advantage in Experiment 3a, equating the $D$ parameter across the cuing conditions did not significantly affect model fit for any of the data sets [Exp. 3a: single-face, $G^{2}(1)=3.383, p=.067$; multiple-face, $G^{2}(1)=1.658, p=.194$; Exp. 3b: $\left.G^{2}(1)=0.124, p=.725\right]$.

Results summary The overall source data replicated results from Experiment 1: Providing face cues improved source discriminability in the multiple-face condition, but not in the singleface condition. The cuing effects were attributed to changes in source memory by both a bivariate signal detection model and a

Table $6 G^{2}$ and parameter values for the multinomial model results

\begin{tabular}{|c|c|c|c|c|c|c|c|c|c|c|c|}
\hline Data Set & $G^{2}(2)$ & $D_{\mathrm{C}}$ & $D_{\mathrm{NC}}$ & $d_{\mathrm{C}}$ & $d_{\mathrm{NC}}$ & $b_{\mathrm{C}}$ & $b_{\mathrm{NC}}$ & $a_{\mathrm{C}}$ & $a_{\mathrm{NC}}$ & $g_{\mathrm{C}}$ & $g_{\mathrm{NC}}$ \\
\hline Experiment $3 \mathrm{a}$, Single-face & 2.057 & .64 & .60 & .81 & .81 & .48 & .39 & .35 & .46 & .62 & .51 \\
\hline Experiment 3a, Multiple-face & 1.385 & .56 & .53 & .81 & .68 & .48 & .29 & .53 & .55 & .47 & .57 \\
\hline Experiment 3b (Multiple-face) & 1.599 & .64 & .65 & .83 & .72 & .60 & .31 & .26 & .47 & .54 & .62 \\
\hline
\end{tabular}

The subscript "C" denotes cued trials, and "NC" denotes trials with no cue. $D=$ probability of item detection; $d=$ probability of source detection; $b=$ probability of guessing "old"; $a=$ probability of guessing "male" for items detected as old; $g=$ probability of guessing "male" for items called "old" on the basis of guessing 
multinomial model. For the multiple-face participants, cues also made recognition responding more liberal, resulting in a higher false-alarm rate for cued than for uncued trials. This liberal shift lowered conditionalized source accuracy for the cued condition, counteracting the benefit to source memory.

Some might conclude that cues only improved source memory for items that were called "new," given that there was no difference in source accuracy for items called "old" in the cued and uncued conditions. This is not a correct interpretation, as it fails to consider the impact of recognition biases on conditionalized source accuracy (DeCarlo, 2003). Our results indicate that cues improved source memory even for items that were called "old." Perhaps the best way to understand this result is to consider the multinomial modeling. These models are usually applied to tasks in which participants only make a source judgment after an "old" response. Accordingly, we fit our models to the frequency of "male" and "female" judgments only for items that were called "old." The parameters showed a cuing benefit on source detection for these items, demonstrating that cues improved source memory for recognized items.

The multinomial results also provide a nice way to demonstrate how recognition biases offset the cuing advantage for conditionalized source accuracy. Matching the empirical results, the predicted values from the multinomial models showed little or no change in conditionalized source accuracy between cued and uncued trials. For example, consider the data and predictions from Experiment 3b. In Table 5, the conditionalized source accuracy for male items is reported in the " $M$ " $\mid M$ column, and the conditionalized source accuracy for female items is 1 minus " $M$ " $\mid$ F. These values range between .79 and .82, with no hint of a cuing effect. Crucially, the model from Experiment $3 \mathrm{~b}$ predicted the same pattern even though the source detection parameter was higher for cued than for uncued trials in this model. Specifically, the predicted conditionalized source accuracy was .81 both with and without cues. In other words, little or no effect on conditionalized source accuracy is exactly the result one should expect even if cues increased source memory for items called "old." If there were no cuing effects on source memory, then conditionalized source accuracy should have been lower in the cued condition as a result of the increase in guessing "old" for undetected items.

The results provided some evidence that cues improved recognition memory, but the effects were small and inconsistent. Dodson and Shimamura (2000) found no impact of cues from the study phase on item memory, ${ }^{2}$ and Vogt and

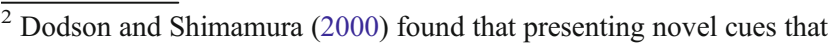
were not presented in the study phase slightly impaired item memory relative to familiar cues that were presented at encoding, but they reported no cuing effects on item memory between familiar cues and uncued trials, and no difference between matching cues (same voice studied with the item) and familiar mismatching cues (voice studied for other studied items, but not the test item). We did not have any conditions with novel cues.
}

Bröder (2007) accommodated their data with models that used the same item-detection parameter regardless of cuing condition. Given these studies and our own results, we conclude that item memory effects are either quite small (and thus commonly go undetected) or nonexistent (with a few false positives). This suggests that information from one specific source dimension (e.g., gender) plays a relatively small role in recognition judgments. Our interest is the effect of cues on source memory, so the critical results are from the judgments that specifically target source information. The source data clearly showed cuing effects in the multiple-face conditions, as expected.

\section{General discussion}

Our experiments provided strong support for the internalcuing account. In Experiment 1, we compared cuing effects in groups that studied a single male and female face to groups that studied multiple male and female faces. As predicted by the internal-cuing account, external cues significantly improved source discrimination for the multiple-face participants but not the single-face participants. Experiment 2 demonstrated that facilitating internal reinstatement at test eliminated cuing effects in the multiple-face condition. Finally, Experiments $3 \mathrm{a}$ and $3 \mathrm{~b}$ validated the conclusions from Experiment 1 with a joint recognition/source-memory test. These experiments also showed that multinomial modeling supports the same conclusions about source memory as the $d^{\prime}$ measures based on a signal detection model.

Our procedure of presenting cues from both the male and female source differs from previous cuing studies, in which cues were always specific to a single source (Craik \& Kirsner, 1974; Dodson \& Shimamura, 2000; Kirsner, 1973; Leynes et al., 2003; Palmeri et al., 1993; Roediger et al., 2004; Starns \& Hicks, 2005; Vogt \& Bröder, 2007). As a consequence, our results might not be directly comparable with past work. However, our results do indicate that internal reinstatement is a critical factor in determining cue effectiveness. We found effect sizes in the very small to small range in conditions designed to support accurate internal reinstatement, as compared to effect sizes in the medium to large range in conditions designed to impair internal reinstatement. The equivocal cuing results in the past literature are not surprising, given that these studies used encoding procedures similar to our single-face conditions.

Cuing investigations promise to enhance our understanding of the processes involved in source decisions. The present results suggest that source decisions require some form of internal reinstatement. In Smith's (1979) recall experiment, participants had to be specifically instructed to internally reinstate the learning context. With a source task, participants seem to internally reinstate context even without special instructions. As such, our results are consistent with an early-selection view 
of source memory, whereby participants constrain memory retrieval by reinstating encoding processes at test (Jacoby, Kelley, \& McElree, 1999; Jacoby, Shimizu, Daniels, \& Rhodes, 2005). For example, to selectively search for evidence that a word was studied in a male voice, one could internally reinstate details of the male voice heard at encoding and evaluate the mnemonic evidence produced by this cue.

This sort of retrieval process is also suggested by global matching models of memory. In these models, evidence that an item was studied in a particular source can be obtained by interactively probing memory with the current test item and characteristics of the source (Hicks \& Starns, 2006; Hintzman, 1988; Humphreys, Bain, \& Pike, 1989; Ratcliff, Van Zandt, \& McKoon, 1995). Notably the bind-cue-decide model of episodic memory (BCDMEM) explicitly proposes that recognition and source judgments require internally reinstated context information (Dennis \& Humphreys, 2001). The reinstated context in BCDMEM is similar to the notion of a source schema or template in the source-monitoring framework (SMF; Johnson et al., 1993); that is, a general representation of the types of memory evidence that would be expected for a memory that originated in a particular source. Proponents of the SMF have emphasized that source schemas can determine the nature of what is activated and/or evaluated in memory traces (Lindsay \& Johnson, 1989; Marsh \& Hicks, 1998; Starns, Lane, Alonzo, \& Roussel, 2007), and cues could improve performance by guiding the formation of more appropriate source schemas.

Murnane, Phelps, and Malmberg (1999) developed the item-context-ensemble (ICE) model to explain the presence or absence of context reinstatement effects on recognition discriminability. The model proposes that discriminability effects will only be observed when participants encode integrated representations that include both context and item details ("ensemble" representations), and the authors report results consistent with that claim (see also Craik \& Schloerscheidt, 2011). We instructed people to encode items by imagining the person in the picture demonstrating the meaning of the presented word, so participants in all of our conditions should have formed ensemble representations at encoding. As such, our results show that internal reinstatement can eliminate cuing effects even when ensemble information is available. This is most poignantly demonstrated in Experiment 2, where structuring the test to facilitate internal reinstatement eliminated cuing effects following the same encoding conditions that produced ample cuing effects when internal reinstatement was difficult.

\section{Conclusion}

Many otherwise diverse theories of memory share a common emphasis on the importance of retrieval cues (see Roediger,
2000). In general, theorists agree that memory should be most successful when the cues available at retrieval closely correspond to the characteristics of the target event (e.g., Tulving \& Thomson, 1973). Nevertheless, a number of studies have found null effects of experimenter-provided cues on source memory (Craik \& Kirsner, 1974; Kirsner, 1973; Starns \& Hicks, 2005; Vogt \& Bröder, 2007). The present study provides evidence that retrieval cues do affect source performance, but participants are not limited to the external cues manipulated by the experimenter. Ironically, source reinstatement manipulations can fail because effective source cues are so important that participants internally reinstate them even when they are not explicitly prompted to do so.

\section{Appendix}

Here, we describe our methodology for applying a multinomial model to the recognition and source data from Experiments 3a and $3 \mathrm{~b}$. Multinomial models assume that responses are determined by discrete underlying states and that the model parameters estimate the probability of entering each state (Batchelder $\&$ Riefer, 1990). For the recognition decision, item detection succeeds with probability $D$, leading to a correct response. When item detection fails, participants guess "old" with probability $b$ or "new" with probability $(1-b)$. Following successful item detection, source detection succeeds with probability $d$, and participants make an accurate source judgment. When source detection fails, participants guess "male" with a proportion estimated by one of two parameters: $a$ reflects source guessing when item detection succeeds and source detection fails, whereas $g$ reflects source guessing when both item and source detection fail, and the participant guesses "old."

We prepared our data in the traditional way for multinomial modeling. For each condition and item type, we computed the frequencies of "old-male," "old-female," and "new" responses. We did not segment the "new" responses for studied items on the basis of the response for the source judgment. Thus, there were two degrees of freedom in the data for each condition (one of the three response frequencies was constrained because they must sum to the total number of trials). We ran separate fits for the multiple-face and singleface groups from Experiments $3 \mathrm{a}$ and $3 \mathrm{~b}$. Each fit had six conditions - male, female, and new items with and without cues - producing 12 degrees of freedom in the data.

The full multinomial model for this data set had eight free parameters for each cuing condition: the item detection $(D)$ parameter for male, female, and new items; the source detection $(d)$ parameter for male and female items; the item guessing parameter $(b)$; and the two source guessing parameters $(a$ and $g$ ). Because this full model had more free parameters than there were degrees of freedom in the data, the parameter space had to be constrained in some way. Following Dodson 
and Shimamura (2000), we equated the item and source detection parameters across all item types (Vogt \& Bröder, 2007, also used this strategy for all of their source dimensions except font size). This eliminated six free parameters, resulting in a tenparameter model. This model provided a close fit to each data set (maximum $G^{2}$ of 2.06 , as compared with a critical value of 5.99 for model rejection at the .05 alpha level, $d f=2$ ).

We conducted $G^{2}$ tests to evaluate the potential cuing effects for item and source memory (Batchelder \& Riefer, 1990). Specifically, we equated either the item detection, item bias, or source detection parameter across cued and uncued trials, and evaluated how much this inflated the $G^{2}$ value. The test statistic in this case was the difference in $G^{2}$ between the tenparameter model and the constrained model, and this statistic was asymptotically distributed as a $\chi^{2}$ distribution with one degree of freedom. (These tests are reported in the main text of the article.) Table 6 reports the best-fitting parameter and $G^{2}$ values from all of our fits.

\section{References}

Banks, W. P. (2000). Recognition and source memory as multivariate decision processes. Psychological Science, 11, 267-273.

Batchelder, W. H., \& Riefer, D. M. (1990). Multinomial processing models of source monitoring. Psychological Review, 97, 548-564. doi:10.1037/0033-295X.97.4.548

Bayen, U. J., Murnane, K., \& Erdfelder, E. (1996). Source discrimination, item detection, and multinomial models of source monitoring. Journal of Experimental Psychology: Learning, Memory, and Cognition, 22, 197-215. doi:10.1037/0278-7393.22.1.197

Bjork, R. A., \& Richardson-Klavehn, A. (1989). On the puzzling relationship between environmental context and human memory. In C. Izawa (Ed.), Current issues in cognitive processes: The Tulane Flowerree Symposium on Cognition (pp. 313-344). Hillsdale: Erlbaum.

Bröder, A., \& Meiser, T. (2007). Measuring source memory. Journal of Psychology, 215, 52-60. doi:10.1027/0044-3409.215.1.52

Clark, S. E., \& Gronlund, S. D. (1996). Global matching models of recognition memory: How the models match the data. Psychonomic Bulletin \& Review, 3, 37-60. doi:10.3758/BF03210740

Cohen, J. (1988). Statistical power analysis for the behavioral sciences (2nd ed.). Hillsdale: Erlbaum.

Craik, F. I. M., \& Kirsner, K. (1974). The effect of speaker's voice on word recognition. Quarterly Journal of Experimental Psychology, 26, 274-284.

Craik, F. I. M., \& Schloerscheidt, A. M. (2011). Age-related differences in recognition memory: Effects of materials and context change. Psychology and Aging, 26, 671-677.

DeCarlo, L. T. (2003). Source monitoring and multivariate signal detection theory, with a model for selection. Journal of Mathematical Psychology, 47, 292-303. doi:10.1016/S0022-2496(03)00005-1

Dennis, S., \& Humphreys, M. S. (2001). A context noise model of episodic word recognition. Psychological Review, 108, 452-478. doi:10.1037/0033-295X.108.2.452

Dodson, C. S. (2007). Retrieval-based illusory recollections: Why study-test contextual changes impair source memory. Memory \& Cognition, 35, 1211-1221

Dodson, C. S., \& Shimamura, A. P. (2000). Differential effects of cue dependency on item and source memory. Journal of Experimental
Psychology: Learning, Memory, and Cognition, 26, 1023-1044. doi:10.1037/0278-7393.26.4.1023

Dube, C., \& Rotello, C. M. (2012). Binary ROCs in perception and recognition memory are curved. Journal of Experimental Psychology: Learning, Memory, and Cognition, 38, 130-151. doi:10.1037/ a0024957

Dube, C., Rotello, C. M., \& Heit, E. (2010). Assessing the belief bias effect with ROCs: It's a response bias effect. Psychological Review, 117, 831-863.

Dube, C., Starns, J. J., Rotello, C. M., \& Ratcliff, R. (2012). Beyond ROC curvature: Strength effects and response time data support continuous-evidence models of recognition memory. Journal of Memory and Language, 67, 389-406.

Erdfelder, E., \& Buchner, A. (1998). Decomposing the hindsight bias: A multinomial processing tree model for separating recollection and reconstruction in hindsight. Journal of Experimental Psychology: Learning, Memory, and Cognition, 24, 387-414.

Fernandez, A., \& Glenberg, A. M. (1985). Changing environmental context does not reliably affect memory. Memory \& Cognition, 13, 333-345. doi:10.3758/BF03202501

Godden, D., \& Baddeley, A. (1980). When does context influence recognition memory? British Journal of Psychology, 71, 99-104. doi:10.1111/j.2044-8295.1980.tb02735.x

Hicks, J. L., \& Starns, J. J. (2006). Remembering source evidence from associatively related items: Explanations from a global matching model. Journal of Experimental Psychology: Learning, Memory, and Cognition, 32, 1164-1173.

Hintzman, D. L. (1988). Judgments of frequency and recognition memory in a multiple-trace memory model. Psychological Review, 95, 528-551. doi:10.1037/0033-295X.95.4.528

Humphreys, M. S., Bain, J. D., \& Pike, R. (1989). Different ways to cue a coherent memory system: A theory for episodic, semantic, and procedural tasks. Psychological Review, 96, 208-233. doi:10.1037/0033-295X.96.2.208

Jacoby, L. L., Kelley, C. M., \& McElree, B. D. (1999). The role of cognitive control: Early selection versus late correction. In $\mathrm{S}$. Chaiken \& Y. Trope (Eds.), Dual-process theories in social psychology (pp. 383-400). New York: Guilford Press.

Jacoby, L. L., Shimizu, Y., Daniels, K. A., \& Rhodes, M. G. (2005). Modes of cognitive control in recognition and source memory: Depth of retrieval. Psychonomic Bulletin \& Review, 12, 852-857. doi:10.3758/BF03196776

Johnson, M. K., Hashtroudi, S., \& Lindsay, D. S. (1993). Source monitoring. Psychological Bulletin, 114, 3-28. doi:10.1037/ 0033-2909.114.1.3

Kirsner, K. (1973). An analysis of the visual component in recognition memory for verbal stimuli. Memory \& Cognition, 1, 449-453.

Klauer, K. C., \& Kellen, D. (2010). Toward a complete decision model of item and source recognition: A discrete-state approach. Psychonomic Bulletin \& Review, 17, 465-478. doi:10.3758/PBR.17.4.465

Leynes, P. A., Bink, M. L., Marsh, R. L., Allen, J. D., \& May, J. C. (2003). Test modality affects source monitoring and eventrelated potentials. The American Journal of Psychology, 116, 389-413.

Lindsay, D. S., \& Johnson, M. K. (1989). The eyewitness suggestibility effect and memory for source. Memory \& Cognition, 17, 349-358.

Malmberg, K. J. (2002). On the form of ROCs constructed from confidence ratings. Journal of Experimental Psychology: Learning, Memory, and Cognition, 28, 380-387.

Maner, J. K., Kenrick, D. T., Becker, D. V., Delton, A. W., Hofer, B., Wilbur, C. J., et al. (2003). Sexually selective cognition: Beauty captures the mind of the beholder. Journal of Personality and Social Psychology, 85, 1107-1120.

Marsh, R. L., \& Hicks, J. L. (1998). Test formats change source-monitoring decision processes. Journal of Experimental Psychology: Learning, Memory, and Cognition, 24, 1137-1151. 
Martin, C. S., Mullennix, J. W., Pisoni, D. B., \& Summers, W. V. (1989). Effects of talker variability on recall of spoken word lists. Journal of Experimental Psychology: Learning, Memory, and Cognition, 15, 676-684.

Murnane, K., \& Bayen, U. J. (1996). An evaluation of empirical measures of source identification. Memory \& Cognition, 24, 417-428. doi:10.3758/BF03200931

Murnane, K., \& Phelps, M. P. (1993). A global activation approach to the effect of changes in environmental context on recognition. Journal of Experimental Psychology: Learning, Memory, and Cognition, 19, 882-894. doi:10.1037/0278-7393.19.4.882

Murnane, K., \& Phelps, M. P. (1994). When does a different environmental context make a difference in recognition? A global activation model. Memory \& Cognition, 22, 584-590.

Murnane, K., \& Phelps, M. P. (1995). Effects of changes in relative cue strength on context-dependent recognition. Journal of Experimental Psychology: Learning, Memory, and Cognition, 21, 158-172. doi:10.1037/0278-7393.21.1.158

Murnane, K., Phelps, M. P., \& Malmberg, K. (1999). Context-dependent recognition memory: The ICE theory. Journal of Experimental Psychology. General, 128, 403-415. doi:10.1037/0096-3445.128.4.403

Palmeri, T. J., Goldinger, S. D., \& Pisoni, D. B. (1993). Episodic encoding of voice attributes and recognition memory for spoken words. Journal of Experimental Psychology: Learning, Memory, and Cognition, 19, 309-328. doi:10.1037/0278-7393.19.2.309

Ratcliff, R., Sheu, C., \& Gronlund, S. D. (1992). Testing global memory models using ROC curves. Psychological Review, 99, 518-535. doi:10.1037/0033-295X.99.3.518

Ratcliff, R., Van Zandt, T., \& McKoon, G. (1995). Process dissociation, single-process theories, and recognition memory. Journal of Experimental Psychology. General, 124, 352-374. doi:10.1037/ 0096-3445.124.4.352

Roediger, H. L., III. (2000). Why retrieval is the key process in understanding human memory. In E. Tulving (Ed.), Memory, consciousness, and the brain: The Tallinn Conference (pp. 52-75). New York: Psychology Press.

Roediger, H. L., III, McDermott, K. B., Pisoni, D. B., \& Gallo, D. A. (2004). Illusory recollection of voices. Memory, 12, 586-602. doi:10.1080/09658210344000125

Schütz, J., \& Bröder, A. (2011). Signal detection and threshold models of source memory. Experimental Psychology, 58, 293-311.

Slotnick, S. D., \& Dodson, C. S. (2005). Support for a continuous (single-process) model of recognition memory and source memory. Memory \& Cognition, 33, 151-170. doi:10.3758/BF03195305
Smith, S. M. (1979). Remembering in and out of context. Journal of Experimental Psychology: Human Learning and Memory, 5, 460471. doi:10.1037/0278-7393.5.5.460

Smith, S. M., Glenberg, A., \& Bjork, R. A. (1978). Environmental context and human memory. Memory \& Cognition, 6, 342-353. doi:10.3758/BF03197465

Smith, S. M., \& Vela, E. (2001). Environmental context-dependent memory: A review and meta-analysis. Psychonomic Bulletin \& Review, 8, 203-220. doi:10.3758/BF03196157

Snodgrass, J. G., \& Corwin, J. (1988). Pragmatics of measuring recognition memory: Applications to dementia and amnesia. Journal of Experimental Psychology. General, 117, 34-50. doi:10.1037/ 0096-3445.117.1.34

Starns, J. J., \& Hicks, J. L. (2005). Source dimensions are retrieved independently in multidimensional monitoring tasks. Journal of Experimental Psychology: Learning, Memory, and Cognition, 31, 1213-1220. doi:10.1037/0278-7393.31.6.1213

Starns, J. J., \& Hicks, J. L. (2008). Context attributes in memory are bound to item information, but not to one another. Psychonomic Bulletin \& Review, 15, 309-314. doi:10.3758/PBR.15.2.309

Starns, J. J., Hicks, J. L., Brown, N. L., \& Martin, B. A. (2008). Source memory for unrecognized items: Predictions from multivariate signal detection theory. Memory \& Cognition, 36, 1-8. doi:10.3758/ MC.36.1.1

Starns, J. J., Lane, S. M., Alonzo, J. D., \& Roussel, C. C. (2007). Metamnemonic control over the discriminability of memory evidence: A signal detection analysis of warning effects in the associative list paradigm. Journal of Memory and Language, 56, 592-607.

Tulving, E., \& Thomson, D. M. (1973). Encoding specificity and retrieval processes in episodic memory. Psychological Review, 80, 352-373. doi:10.1037/h0020071

Vogt, V., \& Bröder, A. (2007). Independent retrieval of source dimensions: An extension of results by Starns and Hicks (2005) and a comment on the ACSIM measure. Journal of Experimental Psychology: Learning, Memory, and Cognition, 33, 443-450.

Watkins, O. C., \& Watkins, M. J. (1975). Buildup of proactive inhibition as a cue-overload effect. Journal of Experimental Psychology: Human Learning and Memory, 1, 442-452. doi:10.1037/02787393.1.4.442

Wixted, J. T. (2007). Dual-process theory and signal-detection theory of recognition memory. Psychological Review, 114, 152-176. doi:10.1037/0033-295X.114.1.152 